\title{
Research Paper: Effects of Gallium Arsenide Laser on Pain and Grip Strength in Patients With Tennis Elbow
}

\author{
Gholamreza Olyaei ${ }^{1}$, Siamak Bashardoust Tajali $^{2 *}$, Behrouz Ataye Mansouri ${ }^{3}$, Reza Shafipoor ${ }^{4}$ \\ 1. Professor, Department of Physiotherapy, School of Rehabilitation, Tehran University of Medical Sciences, Tehran, Iran \\ 2. Assistant Professor, Department of Physiotherapy, School of Rehabilitation, Tehran University of Medical Sciences, Tehran, Iran. \\ 3. PhD, Department of Physiotherapy, School of Rehabilitation, Tehran University of Medical Sciences, Tehran, Iran. \\ 4. Assistant Professor, Department of Orthopedics, School of Medicine, Lorestan University of Medical Sciences, Khorramabad, Iran.
}

Article info:

Received: 08 Aug. 2016

Accepted: 30 Nov. 2016

Critation: Olyaei Gh, Bashardoust Tajali S, Ataye Mansouri B, Shafipoor R. Effects of Gallium Arsenide Laser on Pain and Grip Strength in Patients With Tennis Elbow. Journal of Modern Rehabilitation. 2017; 11(1):45-50. https://doi.org/10.18869/ nirp.jmr.11.1.45

https://doi.org/10.18869/nirp.jmr.11.1.45

\section{Keywords:}

High power laser, Ga-As laser, Tennis elbow, Pain, Grip strength

\section{ABSTRACT}

Introduction: Tennis elbow (Lateral Epicondylitis) is one the most common elbow and arm musculoskeletal disorders. About $1 \%$ to $3 \%$ of active population in modern societies suffer from tennis elbow. Tennis elbow is usually caused by repetitive activities of hand and wrist, especially wrist extension and forearm supination. This research aimed to study high and also medium power gallium arsenide (Ga-As) laser irradiation on improving pain and grip strength (functional mobility) in patients with tennis elbow.

Materials and Methods: Thirty female [Mean(SD) age: 49(2.3) y] patients with acute tennis elbow were recruited for this study. The patients were randomly divided into two groups based on random allocation table. Group A (15 patients) had been received $10 \mathrm{~J} / \mathrm{cm}^{2}$ under a $0.5 \mathrm{~W}$ beam medium power Ga-As laser irradiation of $980 \mathrm{~nm}$ wave length. Group B (15 patients) had been received same energy density under $2 \mathrm{~W}$ beam high Ga-As laser irradiation with the same wavelength. Laser irradiation was performed on a $9 \mathrm{~cm}^{2}$ surface of lateral elbow epicondyle based on Grid laser application technique. The patients were under treatment for 10 sessions every other day. The outcome measures of pain at rest and ring finger extension against loading, grip strength and hand function were recorded and analyzed in a pre- and post-setting. The pain severity was measured by VAS (Visual Analog Scale) and McGill questionnaire and upper extremity function was measured by DASH questionnaire.

Results: Significant pain reduction was observed in resting position and ring finger extension against loading after application of both high and medium power $(980 \mathrm{~nm}, 0.5-2 \mathrm{~W})$ laser therapy $(\mathrm{P}<0.05)$ for 10 sessions. High power laser irradiation could also increase grip strength and mobility in patients with lateral epicondylitis $(\mathrm{P}<0.05)$.

Conclusion: High and low power Ga-As laser irradiation may improve pain and grip strength in patients with acute tennis elbow.

* Corresponding Author: 


\section{Introduction}

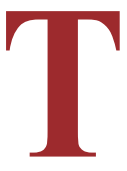

ennis elbow (Lateral epicondylitis) is one of the common elbow and arm musculoskeletal disorders $[1,2]$ that affect $1 \%$ to $3 \%$ of active population among modern societies [3]. Runge, a German physician, was usually credited for the first description of the condition as "writer's cramp" (Schreibekrampf) in 1873 [47]. He was the first person identified and explained main characteristic of this musculoskeletal disorder as incapability of writing $[8,9]$. Later, Henry Morris, a British surgeon, called it tennis elbow in 1883 [10]. Tennis elbow is commonly attributed to tennis players, but it has also been identified among housewives and those who carry heavy things by their hands [3-6]. Generally, arm and elbow joints are under pressure in many occupations such as painting, plumbing, carpentry, car mechanics, cooking and boning. Although less than $5 \%$ to $10 \%$ of patients with tennis elbow are tennis players, more than $50 \%$ of the tennis players may experience this illness in their lifetimes [7]. This syndrome is more common in women generally those between 30 to 60 years $[3,5,6,8]$.

Recent electromyography studies indicate that the origin of Extensor Carpi Radialis Brevis (ECRB) muscle is commonly damaged more than other extensor muscles. Moreover, its repair process is slow and difficult following the injury $[3,11,12]$. Pathophysiologic studies identified an increase of fibroblasts number and levels of proto and amino glycoprotein and also vascular hyperplasia in damaged area $[13,14]$. These patients feel gradual pain with seizures at the lateral side of their elbows. The pain gradually increases and worsens by object pressing, lifting, weaving, carpet-making and in forearm pronation. In chronic cases, pain is felt around forearm and wrist extensor muscles $[13,14]$. The symptoms usually affect activities of daily living [4]. Differential diagnostic maneuvers are generally followed by increased pain even in lateral epicondyle touching, wrist and middle finger extension strength and passive wrist flexion movements [5].

More than 40 treatment protocols have been suggested for tennis elbow such as deep massage, manipulation, steroidal or non-steroidal injections, shockwave, low or high level laser irradiation, as well as brace or therapeutic exercises. However, there is still no approved evidence-based treatment method for lateral epicondylitis $[3,5,15]$. One of the widely-suggested treatments which has been used in past two decades leading to reduce pain in musculoskeletal disorders with various etiologies is Low Level Laser Therapy (LLLT) [16]. Physiotherapists apply the LLLTs in different cases such as carpal tunnel syndrome, joint disorders, tendinopathy, medial/lateral epicondylitis, osteoarthritis, back pain, ankle sprains, venous ulcers, and diabetes [16]. Low level or cold laser therapy with a wavelength of $904 \mathrm{~nm}$ has less than 500 $\mathrm{mW}$ power and high level laser with similar wavelength has a power higher than $500 \mathrm{~mW}$. The effectiveness penetration depth for high power lasers is about 3 to $4 \mathrm{~cm}$ [17]. The laser biological effects depend on three important parameters: wavelength $(\mathrm{nm})$, wave density $\left(\mathrm{j} / \mathrm{cm}^{2}\right)$ and wave intensity (W). Higher wavelengths and output powers increase laser penetration depth [18-20]. Former studies have shown that patients with lateral epicondylitis often had trouble in their jobs, so that they might leave their work for a long period. The amount of pain in these people is usually high, and in later stages they may have trouble even in their social life [4].

This study aimed to compare the effects of gallium arsenide laser with $980 \mathrm{~nm}$ wavelength and output powers of 0.5 and $2 \mathrm{~W}$ (low and high levels) on pain and grip strength in patients with tennis elbow (at rest position and during middle finger extension strength movement). Possible suggested methods and/or dosages lead helping to reduce pain level or improve function in patients with tennis elbow will be an efficient care for these damaged people. Impact of low and high level laser at a specific wavelength has not been evaluated in patients with tennis elbow yet. Also, effective dosages or powers may shorten treatment process and help the patients come back to ordinary life sooner.

\section{Materials and Methods}

Thirty-six female carpet weavers [Mean(SD) age: 49(2.3)] with confirmed diagnosis of acute tennis elbow by an orthopedist or neurosurgeon were recruited for this study. The inclusion criteria comprised aged between 40 and 60 years, having one-sided pain or high sensation of lateral aspect of elbow, feeling increased pain on elbow extension or pronation against pressure, having symptoms started at least 14 days up to 60 days of initial crisis, not having statistical changes of nerve conduction velocity. The exclusion criteria were any signs or symptoms of arthritis, neurologic syndrome, disk herniation, thoracic outlet syndrome, bilateral epicondylitis, radicular pain, carpal tunnel syndrome, tumor, affective medical drugs (NSAIDS or narcotic) or any accompanied musculoskeletal disorders at shoulder or neck.

\section{Assessment of Pain}

Levels of pain were assessed at rest position and during middle finger extension by Visual Analog Scale (VAS) 
and McGill Pain Questionnaire (MPQ). The VAS is a $100 \mathrm{~mm}$ self-measure tool demonstrating levels of pain base on numeric numbers from 0 (no pain) to 10 (highest degree of pain) [21]. After a brief presentation, the patients were asked to sit on a comfortable chair while their elbow and hand were supported next to the body at 90 degrees elbow flexion and wrist neutral position by two pillows. In the next stage, a blinded examiner to the groups asked the patients to identify levels of their pain at rest position and during middle finger extension against small pressure while their finger were located passively on flexion and then they were asked to bring their middle finger to the extension as much as they can. Patients were asked to mark the appropriate score based on their present sense that represents levels of pain at rest and then through middle finger extension against a small pressure. The VAS measures were performed before any intervention and exactly after laser sessions. MPQ is a high reliable valid pain assessment method. It is a self-report questionnaire that allows individuals to give a good description of quality and intensity of their pain. It was designed to assess pain from multiple dimensions [22].

\section{Assessment of grip strength}

The grip strength was measured by a calibrated dynamometer three times with an interval of 30 seconds rest, then the mean score was considered as the grip strength. The grip strength levels were measured before intervention and at the end of laser application sessions.

\section{Assessment of hand function}

The patients were also asked to respond to the Disabilities of Shoulder, Elbow, and Hand (DASH) questionnaire to determine their levels of hand function after treatment sessions. The DASH questionnaire is a reliable valid tool help to measure functional disability in patients with upper extremity disorders [23]. Laser irradiation was performed on lateral elbow epicondyle on a surface of $9 \mathrm{~cm}^{2}$. A clear visible transparent (included 9 rectangles each with the surface area of $1 \mathrm{~cm}^{2}$ ) with a total area of $9 \mathrm{~cm}^{2}$ was designed for laser application to control levels of irradiated energy. The center of each rectangle was empty and the laser beam was emitted at the center of each square for a defined period. The wavelengths of both laser applications were $980 \mathrm{~nm}$ and the exposure time for low level laser $(0.5 \mathrm{~W}$ output power) was 20 seconds and for high level laser was $(2 \mathrm{~W}$ output power) 5 seconds per square. The radiation time for low level laser $(0.5 \mathrm{~W})$ was 180 seconds and for high level laser $(2 \mathrm{~W})$ was 45 seconds. As a result, the amount of energy radiated on each rectangle was $10 \mathrm{~J} / \mathrm{cm}^{2}$ and on lateral aspect of elbow epicondyle was $90 \mathrm{~J} / \mathrm{cm}^{2}\left(9 \mathrm{~cm}^{2}\right)$ for each session. The laser application sessions were continued for 10 sessions every other day. Duration of exposure for each group was calculated based on the previous studies $[15,20]$.

The Kolmogorov-Smirnov normality test was performed to check the normal distribution of variables The independent $t$ test was done to analyze significant differences between the variables among low and high level laser groups. Levels of pain at rest and middle finger extension, grip strength, and upper limb function were compared between two independent laser groups. To identify treatment processes (before and after treatment) in each group, the dependent paired t test was performed. The alpha value was considered 0.05 for significant level.

\section{Results}

According to the results of the K-S analysis are presented in Table 1, the obtained values for $\mathrm{z}$ statistics (at the level of $\alpha=0.05$ ) are not significant. As a result, the assumption of normal distribution of the variables is true. On the basis to the distance based of the variable scales, the independent and dependent $t$ tests were performed between and within (inter- and intra-) groups analysis, respectively.

Based on the study results, level of pain at rest and in middle finger extension against small pressure reduced after low or high levels irradiations; however, high power application was more effective on pain reduction. Average pain assessed at rest and after treatment compared to before treatment indicates a significant reduction in both groups $(\mathrm{P}<0.05)$. There was also a significant difference between two groups $(\mathrm{P}<0.05)$ indicating that high power laser application might be more effective to reduce pain levels at rest position or during middle finger extension against small pressure in patients with acute tennis elbow. Besides, ability of grip strength and upper extremity function were improved after low and high level laser applications, while grip strength did not show substantial improvement following low level laser irradiation. The results are presented in Table 2.

The levels of grip strength and upper extremity function after applying the laser with 0.5 and $2 \mathrm{~W}$ output power were also improved compared to initial steps. It was also shown that the mean grip strength and upper extremity function were significantly higher in the group treated with gallium arsenide laser $980 \mathrm{~nm}$ with $2 \mathrm{~W}$ power 
Table 1. Normal distribution of outcome identified measures

\begin{tabular}{ccc}
\hline & Z Statistics & P \\
\hline McGill pain scale & 1.169 & 0.130 \\
VAS pain scale & 0.976 & 0.297 \\
Upper extremity function (DASH) & 1.175 & 0.126 \\
\hline
\end{tabular}

\section{Discussion}

Tennis elbow, a common musculoskeletal disorder, may disrupt personal or official daily tasks. In this study, effects of low and high level laser irradiation on pain intensity, grip strength, and upper extremity function were studied in patients with acute tennis elbow. The results of this study indicate that high level laser application might be an effective modality on tennis elbow patients' recovery that reduce pain at rest $(\mathrm{P}=0.002)$ and in middle finger extension against small pressure $(\mathrm{P}=0.001)$, improve grip strength $(\mathrm{P}=0.001)$ and upper extremity function $(\mathrm{P}=0.005)$. Our results of high level laser effects on acute tennis elbow were consistent with the results of the similar study conducted by Robert et al. [19]. The results of this study also showed low level laser irradiation of $0.5 \mathrm{~W}$ output power improves pain in patients with acute tennis elbow. Our results of low level laser application on tennis elbow are consistent with the results of some previous similar studies [15, 16, 18-20, 24].

Oken et al. reported that low level laser with short-term application had no effect in improving pain since the absorbed energy density was not enough, while long-term irradiation would be much more effective on pain reduction or better grip strength [17]. Based on laser theory, effectiveness of laser irradiation depends on the amount of absorbed energy [17]. This amount of necessary absorbed energy may increase in a period of time during sessions of application. Our results showed that 10 sessions of low level laser application alleviate pain and increase grip strength in patients with acute tennis elbow. We might reach the necessary effective energy sooner by increasing laser output power.

Table 2. Comparing levels of pain (Mean \pm SD) at rest and during middle finger extension against small pressure, grip strength and upper extremity function before and after low and high levels laser irradiation

\begin{tabular}{|c|c|c|c|c|c|c|}
\hline & & Mean $\pm S D$ & Mean $\pm S D$ & & & \\
\hline & & $0.5 \mathrm{~W}$ & Dep. P & $2 W$ & Dep. P & Indep. P \\
\hline Pain at rest & $\begin{array}{l}\text { Before Tx. } \\
\text { After Tx. }\end{array}$ & $\begin{array}{l}3.788 \pm 66.27 \\
6.021 \pm 35.40\end{array}$ & $0.001 *$ & $\begin{array}{l}3.197 \pm 67.87 \\
5.886 \pm 18.31\end{array}$ & $0.000^{*}$ & $0.002 *$ \\
\hline Pain in middle finger ext. & $\begin{array}{l}\text { Before Tx. } \\
\text { After Tx. }\end{array}$ & $\begin{array}{l}3.960 \pm 65.27 \\
4.515 \pm 35.33\end{array}$ & $0.000^{*}$ & $\begin{array}{l}5.294 \pm 69.80 \\
5.604 \pm 22.53\end{array}$ & $0.000^{*}$ & $0.001 *$ \\
\hline Grip strength & $\begin{array}{l}\text { Before Tx. } \\
\text { After Tx. }\end{array}$ & $\begin{array}{l}0.450 \pm 1.53 \\
0.514 \pm 2.48\end{array}$ & 0.067 & $\begin{array}{l}0.770 \pm 1.893 \\
8.790 \pm 3.473\end{array}$ & $0.001^{*}$ & $0.001 *$ \\
\hline Upper extremity function & $\begin{array}{l}\text { Before Tx. } \\
\text { After Tx. }\end{array}$ & $\begin{array}{l}3.378 \pm 38.47 \\
9.985 \pm 59.87\end{array}$ & $0.015^{*}$ & $\begin{array}{c}3.555 \pm 33.27 \\
11.250 \pm 63.47\end{array}$ & $0.022^{*}$ & $0.005^{*}$ \\
\hline
\end{tabular}


The reason of ineffective reports in some previous studies might be attributed to this amount of necessary absorbed energy. Many previous researchers conducted their studies on low level laser application with a wavelength of $904 \mathrm{~nm}$ and dosage between 2.3 and $4 \mathrm{~J} / \mathrm{cm}^{2}$ [17]. In the present study, the applied wavelength was $980 \mathrm{~nm}$ with a dosage of $10 \mathrm{~J} / \mathrm{cm}^{2}$ that increases the level of penetration and energy absorbed density and better effectiveness for laser application. Although it is possible to provide similar rational for high power laser effectiveness, there is a possible limit for amount of absorbed energy effectiveness, as it is reported much higher densities may lead to strongly inhibit cell function [19]. Basford et al. reported that low-power laser had no effect on pain, tenderness and function which might be due to the fact that he studied effects of a low level ND: YAG laser with a low dosage, while the applied laser in our study was gallium arsenide $980 \mathrm{~nm}$ with a dosage of $10 \mathrm{~J} / \mathrm{cm}^{2}$. The type of laser and necessary dosage might be important factors in treatment effectiveness [25].

Biological effects of laser irradiation depend on the wavelength, absorbed energy density, and output intensity [18]. Higher wavelength or output power increases penetration depth and effects of possible laser irradiation. The relevant studies on low and high level gallium arsenide lasers on acute tennis elbow were few with no comparison between low and high levels application. It seems that the laser with $2 \mathrm{~W}$ output power is more effective than $0.5 \mathrm{~W}$ output power to reduce pain, improve grip strength and upper extremity function. Further studies are necessary to find more details in this field.

High and low power Ga-As laser irradiation may alleviate pain and improve grip strength in patients with acute tennis elbow. High level laser application can be more effective on treatment of these patients. It may help repair the tendon injury and make the treatment process faster due to its deeper penetration.

\section{Acknowledgements}

This research was part of doctorate of physical therapy accomplishment thesis (BAM) and was funded by Sensorimotor Rehabilitation Research Center, Tehran University of Medical Sciences.

\section{Conflict of Interest}

The authors declared no conflicts of interest.

\section{References}

[1] Dundar U, Turkmen U, Toktas H, Ulasli AM, Solak O. Effectiveness of high-intensity laser therapy and splinting in lateral epicondylitis: A prospective, randomized, controlled study. Lasers in Medical Science. 2015; 30(3):1097-107. doi: 10.1007/ s10103-015-1716-7

[2] Kaur M, Arora P, Arora L, Kumar S. Efficacy of laser therapy in lateral epicondylitis: A Randomized clinical trial. International Journal of Recent Research and Review, 2014; 7(2): 2532.

[3] Tosti R, Jennings J, Sewards JM. Lateral Epicondylitis of the Elbow. The American Journal of Medicine. 2013; 126(4):357. e1-357.e6. doi: 10.1016/j.amjmed.2012.09.

[4] Solverborn N. Tennis elbow. Scandinavian Journal of Medicine \& Science in Sports. 1997; 7:229-237.

[5] Gellman H. Tennis elbow (lateral epicondilitis). Orthopedic Clinics of North America. 1992; 23(1):75-82. PMID: 1729672

[6] Haker E, Lundeberg T. Is low-energy laser treatment effective in lateral epicondylalgia? Journal of Pain and Symptom Management. 1991; 6(4):241-6. doi: 10.1016/08853924(91)90014-u

[7] Gruchow HW, Pelletier D. An epidemiologic study of tennis elbow. The American Journal of Sports Medicine. 1979 7(4):234-8. doi: 10.1177/036354657900700405

[8] Dimberg L. The prevalence and causation of tennis elbow (lateral humeral epicondylitis) in a population of workers in an engineering industry. Ergonomics. 1987; 30(3):573-9. doi: $10.1080 / 00140138708969746$

[9] Runge F. [Recognition and treatment of writing (German)] BerlKlin Wochenschr. 1873; 10:245.

[10] Jobe FW, Ciccotti MG. Lateral and medial epicondylitis of the elbow. Journal of the American Academy of Orthopaedic Surgeons. 1994; 2(1):1-8. doi: 10.5435/00124635-19940100000001

[11] Morrey BF. The Elbow and its disorders. $4^{\text {th }}$ edition. Amsterdam: Elsevier; 2009.

[12] Verhaar J. Tennis elbow. Anatomical epidemiological and therapeutic aspects. International Orthopaedics. 1994; 18(5). doi: $10.1007 /$ bf00180221

[13] Morris M, Jobe FW, Perry J, Pink M, Healy BS. Electromyographic analysis of elbow function in tennis players. The American Journal of Sports Medicine. 1989; 17(2):241-7. doi: $10.1177 / 036354658901700215$

[14] Pienimaki, T. Conservative treatment and rehabilitation of tennis elbow: A review article. Critical Reviews in Physical and Rehabilitation Medicine. 2000; 12(3):16. doi; 10.1615/critrevphysrehabilmed.v12.i3.20

[15] Simunovic Z, Trobonjaca T, Trobonjaca Z. Treatment of medial and lateral epicondylitis tennis and golfer's elbow with low level laser therapy: A multicenter double blind, placebo-controlled clinical study on 324 patients. Journal of Clinical Laser Medicine \& Surgery. 1998; 16(3):145-51. doi 10.1089/clm.1998.16.145 
[16] Apostolos T. Effects of low-level laser and plyometric exercises in treatment of lateral epicondylitis. Photomedicine and Laser Surgery. 2007; 25(3):205-13. doi: 10.1089/pho.2007.2041

[17] Roberts DB, Kruse RJ, Stoll SF. The effectiveness of therapeutic class IV $(10 \mathrm{~W})$ laser treatment for epicondylitis. Lasers in Surgery and Medicine. 2013; 45(5):311-7. doi: 10.1002/ lsm. 22140

[18] Tuner J, Hode L. The laser therapy handbook. New York: Prima Book; 2004.

[19] Baxter GD. Therapeutic lasers: Theory and practice. London: Churchill Livingstone; 1999.

[20] Vasseljen O, Hoeg N, Kjeldstad B. Low level laser versus placebo in treatment of tennis elbow. Scandinavian Journal of Rehabilitation Medicine. 1992; 24(1):37-42. PMID: 1604260

[21] Hjermstad MJ, Fayers PM, Haugen DF, Caraceni A, Hanks GW, Loge JH, et al. Studies comparing Numerical Rating Scales, Verbal Rating Scales, and Visual Analogue Scales for assessment of pain intensity in adults: A systematic literature review. Journal Pain Symptom Management. 2011; 41(6):1073-93. doi: 10.1016/j.jpainsymman.2010.08.016

[22] urckhardt CS, Jones KD. Adult measures of pain: The McGill Pain Questionnaire (MPQ), Rheumatoid Arthritis Pain Scale (RAPS), Short-Form McGill Pain Questionnaire (SFMPQ), Verbal Descriptive Scale (VDS), Visual Analog Scale (VAS), and West Haven-Yale Multidisciplinary Pain Inventory (WHYMPI). Arthritis \& Rheumatism. 2003; 49(S5):S96S104. doi: $10.1002 /$ art.11440

[23] Raven EEJ, Haverkamp D, Sierevelt IN, Van Montfoort DO, Pöll RG, Blankevoort L, et al. Construct validity and reliability of the disability of arm, shoulder and handquestionnaire for upper extremity complaints in rheumatoid arthritis. Journal of Rheumatoly. 2008; 35(12):2334. doi: 10.3899/jrheum.080067

[24] Emanet Sk, Altan LI, Yurtkuran M. Investigation of the effect of GaAs laser therapy on lateral epicondylitis. Photomedicine and Laser Surgery. 2010; 28(3):397-403. doi: 10.1089/ pho.2009.2555

[25] Basford JR, Sheffield CG, Cieslak KR. Laser therapy: A randomized, controlled trial of the effects of low intensity $\mathrm{Nd}$ : YAG laser irradiation on lateral epicondylitis. Archives of Physical Medicine and Rehabilitation. 2000; 81(11):1504-10. doi: 10.1053/apmr.2000.17812 